\title{
Modelling household responses to energy efficiency interventions via system dynamics and survey data
}

\author{
Stephen Davis* $\quad$ Ian Durbach ${ }^{\dagger}$
}

Received: 4 August 2010; Revised: 1 November 2010; Accepted: 6 November 2010

\begin{abstract}
An application of building a system dynamics model of the way households might respond to interventions aimed at reducing energy consumption (specifically the use of electricity) is described in this paper. A literature review of past research is used to build an initial integrated model of household consumption, and this model is used to generate a small number of research hypotheses about how households possessing different characteristics might react to various types of interventions. These hypotheses are tested using data gathered from an efficiency intervention conducted in a town in the South African Western Cape in which households were able to exchange regular light bulbs for more efficient compact fluorescent lamp light bulbs. Our experiences are (a) that a system dynamics approach proved useful in advancing a non-traditional point of view for which, for historical and economic reasons, data were not abundantly available; (b) that, in areas where traditional models are heavily quantitative, some scepticism to a system dynamics model may be expected; and (c) that a statistical comparison of model results by means of empirical data may be an effective tool in reducing such scepticism.
\end{abstract}

Key words: Energy sector, system dynamics.

\section{Introduction}

The question of how households respond to intervention strategies aimed at reducing energy consumption is receiving increasing attention, particularly as the awareness of the need to curb energy consumption strengthens globally. One of the challenges facing energy researchers is to synthesise the many results obtained from a macro-level modelling of the impacts of interventions on consumption ${ }^{1}$ with the smaller number of studies focussing on psychological models of energy consumption at the level of the individual or household. ${ }^{2}$

\footnotetext{
*Energy Research Centre, University of Cape Town, Private Bag, Rondebosch, 7701, South Africa.

${ }^{\dagger}$ Corresponding author: Department of Statistical Sciences, University of Cape Town, Rondebosch, 7701, South Africa, email: ian.durbach@uct.ac.za

${ }^{1}$ See, for example, Nässén and Holmberg (2009), Laitner (2000), Barker et al. (2007), Barker et al. (2009), Saunders (2000), Berkhout et al. (2000), Grepperud and Rasmussen (2004), and Jaccard and Bataille (2000).

${ }^{2}$ See, for example, Swim et al. (2009), Griskevicius et al. (2008), Gyberg and Palm (2009), Owens and Drifill (2008), Fischer (2008), Abrahamse et al. (2005), and Abrahamse et al. (2007).
} 
The primary aim of the current paper is to describe how combining an operations research (OR) model with observational survey data may help to build a model of responses to energy interventions at the level of the household. Our modelling process uses system dynamics (e.g. Ford, 1999; Morecroft, 2007) to synthesise the findings of previous research into a single coherent model framework. This model is then used to investigate how a household's response to various energy intervention strategies may depend on the attitudes it holds. This investigation, although exploratory in nature, yields a number of insights into consumption behaviour that can be stated precisely in the form of research hypotheses. These research hypotheses are tested against data gathered as part of a compact fluorescent lamp (CFL) exchange programme conducted in Prince Albert, a town in the Karoo region of South Africa; in doing so we evaluate the ability of the system dynamics model to explain actual behaviour.

Our aim in presenting this case study is to contribute to the emerging literature on the applications of operational research in development and to provide an indication of how survey and other observational data may be integrated successfully into a system dynamics model. From the perspective of energy research, the paper highlights the value of a multi-criteria approach to energy modelling — one which explicitly considers the tradeoffs between the multiple conflicting objectives faced by a household when consuming electricity.

The remainder of this paper proceeds as follows. In the next section an overview of the main research project is given. Section 3 contains an overview of the approach used to construct and test the system dynamics model. The model and its attributes are described in Section 4. Section 5 gives the results obtained from the model and describes how research hypotheses were generated from these results. Section 6 describes how survey and consumption data were collected and used to aid the modelling process. Some implications for modelling practice suggested by our experiences are discussed in Section 7, and the paper closes in Section 8.

\section{Overview of the SANERI rebound project}

In energy research as well as in other areas of natural resource management, the rebound effect refers to a phenomenon whereby gains in resource efficiency results in a less than expected reduction in resource usage. The amount of the actual reduction in consumption may be less than the technically feasible reduction, since the increased efficiency lowers the cost of consumption for a good or service. The lower cost then results in an increase in consumption of the good or service in question as it becomes relatively cheaper to use ('direct' rebound) and also increases consumption of other energy-consuming goods and services as disposable income increases ('indirect' rebound). Efficiency measures may also increase consumption through increases in economic growth created by the new technologies ('economy-wide' rebound) (Berkhout et al., 2000). If rebound effects become sufficiently large the introduction of an efficiency measure may have the paradoxical effect of actually increasing overall energy consumption.

The University of Cape Town's Energy Research Centre (ERC), with support from the South African National Energy Research Institute (SANERI), initiated a study that aimed 
to quantify the rebound effect of energy efficiency initiatives in South Africa's residential sector. In addition to measuring the effect, the study aimed to identify the potential to mitigate rebound using awareness initiatives. The approach used was to identify sites, previously untouched by the rollout of a particular technology, and to conduct panel surveys before and after the rollout, in combination with the collection of consumption data, and then to analyse the impact of the intervention on energy consumption patterns. A part of this rebound project aimed to explore the factors influencing household electricity consumption and the likely reaction to interventions of price, awareness and technology. It is this aspect of the project that is described here.

\section{Overview of approach used}

Our modelling approach can be summarised as overlapping phases of model construction, model exploration, and model testing. We began by searching the energy-related literature to identify which aspects of attitude and behaviour had been found to affect household electricity consumption. Over a series of meetings these sources were integrated into a single system dynamics model of household electricity consumption. Following this process, a small group of energy researchers at the ERC were consulted and gave informal feedback on the model. This feedback motivated some minor changes to the initial model.

We then generated a small number of household types (with different preferences) and intervention scenarios, and used the model to evaluate the potential impact of each intervention scenario on each household. These results were reported back to the group and used as a basis for discussion. Some changes were again made to the model at this stage, and the simulation process was repeated. We used the results obtained from this modified model to generate a number of testable research hypotheses about consumption behaviour. These hypotheses related to how households possessing different attitudinal characteristics might respond to various interventions.

At this stage the final survey and consumption data from the real-world intervention were collected and used to evaluate the research hypotheses. These results were again presented to our group and used as a basis for further discussion. It was decided that the survey results did not suggest any necessary modifications and that the model (a) appeared to give a simple, but satisfactory explanation for the observed changes in electricity consumption, and (b) provided a justification for the use of attitudinal information in electricity consumption models. At that stage, and pending new information, the modelling process was brought to an end.

\section{A system dynamics model of electricity consumption}

Figure 1 shows our system dynamics model, constructed using Vensim software (Vensim, 2010). A description of the essential aspects of the model is given below. 


\subsection{The basic consumption model}

Previous literature suggests that households have three main objectives when making decisions about which electricity-consuming goods and services to use (and thus about how much electricity ${ }^{3}$ to consume): ${ }^{4}$

- minimising the cost of consuming the electricity,

- maximising the comfort derived from electricity-consuming appliances, and

- minimising the impact of their electricity consumption on the power system and the environment.

In our model cost is measured by multiplying the number of units of electricity consumed by the unit price of electricity. Comfort and impact are both measured (if only indirectly) by the number of units of electricity consumed. Not all households will accord equal importance to all three objectives; preferences are expressed in terms of goals, which define realistically desired levels of performance on each objective, and weights, which define the relative importance of equal-size differences between the goal and performance levels on each objective (e.g. Belton and Stewart, 2002).

Given a particular set of goals and weights for the three objectives, households typically find an electricity consumption level that comes closest to satisfying their aggregated desires over the three objectives. Differences between current performance and desired goals are given by $\delta_{j}=\max \left\{f_{j}(C)-g_{j}, 0\right\}$ for minimising objectives, and $\delta_{j}=$ $\max \left\{g_{j}-f_{j}(C), 0\right\}$ for maximising objectives, where $\delta_{j}$ is the deviation from the goal $g_{j}$ on objective $j$ and $f_{j}(C)$ is an assessment of current performance on objective $j$. Note that under-achievement with respect to a goal is always positively signed and households are indifferent to any over-achievement beyond the goals.

An equilibrium consumption level $C^{*}$ occurs where the desire to increase consumption (and so gain more comfort) is exactly balanced by the desire to decrease consumption (and so save money and cause less environmental damage), i.e. $w_{\text {comf }} \delta_{\text {comf }}=w_{\text {cost }} \delta_{\text {cost }}+$ $w_{i m p} \delta_{i m p}$, where $w_{j}$ is the weight associated with objective $j$. If the current consumption is below (above) the equilibrium level $C^{*}$, then current consumption is increased (decreased) incrementally until it reaches an equilibrium level.

\subsection{External interventions}

The aim of our model is to examine three broad types of intervention strategies that have been found (at least by some studies) to lead to decreases in household electricity consumption.

\footnotetext{
${ }^{3}$ For clarity, electricity consumption is used to refer to the number of kWh (kilowatt hours) used by a household during a particular time period. Strictly speaking, demand is measured in kW (kilowatts) in the case of electricity. For this paper, electricity demand refers to the demand for electricity-consuming goods and services.

${ }^{4}$ See, for example, Ek and Söderholm (2010), Swim et al. (2009), Griskevicius et al. (2008), Gyberg and Palm (2009), Mehlwana and Qase (1996), Birol and Keppler (2000), Abrahamse et al. (2005), Abrahamse et al. (2007), Caird et al. (2008), Wall and Crosbie (2008), Bladh and Krantz (2008), Fischer (2008), Levine et al. (n.d.), and Owens and Drifill (2008).
} 


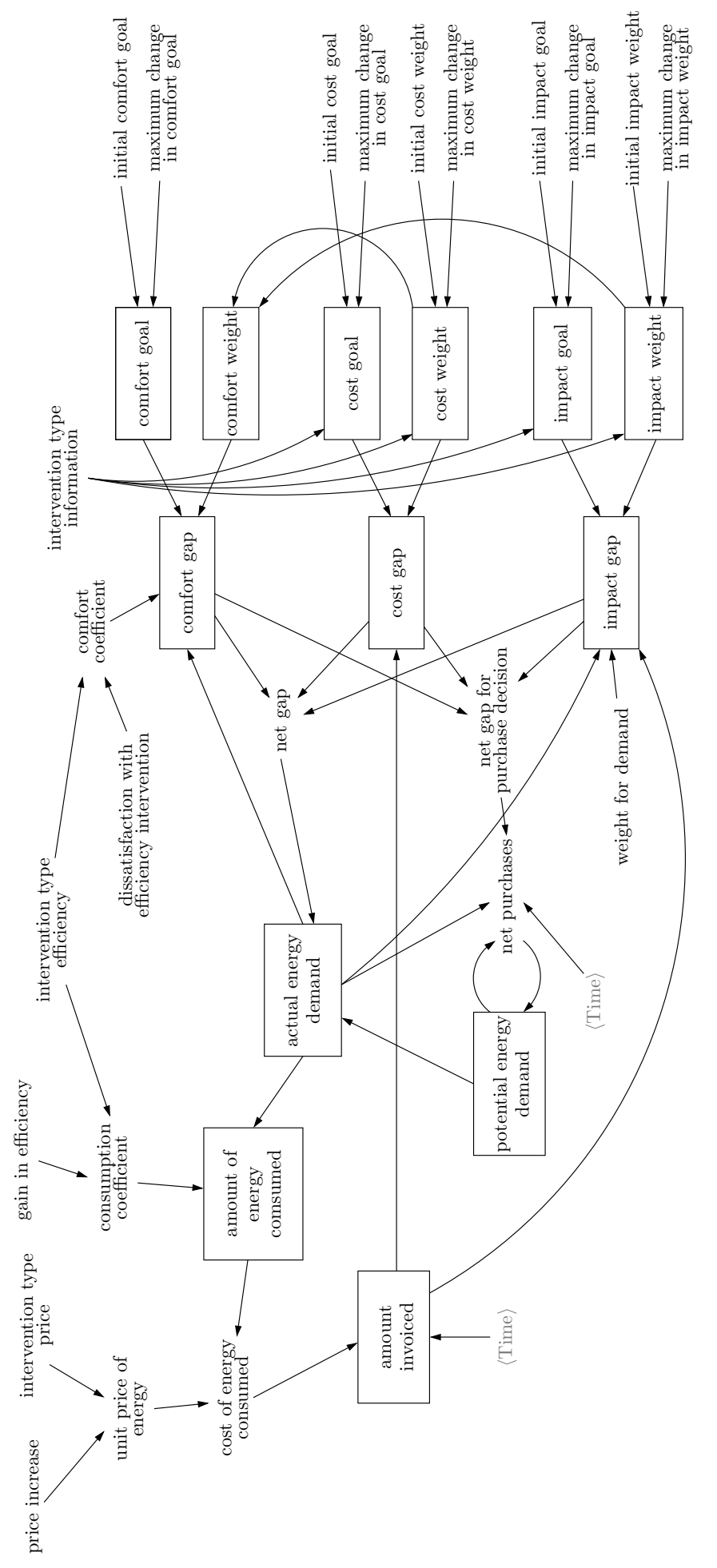

Figure 1: System dynamics model of household energy consumption. 
These three interventions can be categorised as:

1. Price interventions. These attempt to reduce electricity demand by increasing the effective unit price of electricity;

2. Efficiency interventions. These attempt to reduce electricity consumption by introducing new technologies which require less electricity to fulfil the same service (relative to a former, less efficient technology);

3. Information interventions. These attempt to reduce electricity demand by providing households with information about environmental issues related to electricity use, more frequent and effective billing information, or emphasising individual and collective responsibility for sustainable electricity use.

The modelling of each of the interventions is described below.

\subsubsection{Price interventions}

Price increases naturally affect the unit price of electricity, without affecting any other part of the model. If the unit cost of electricity is increased by $(100 \times t) \%$, the new cost incurred by the household is $(1+t) r C$, where $r$ is the per-unit cost of electricity.

\subsubsection{Efficiency interventions}

The introduction of a new, more-efficient technology decreases the amount of electricity required by a household in order to perform some activity. From the household's point of view, the same basic activity is being performed, but less electricity is being used doing so. It is therefore necessary to make a distinction between what might be termed the amount of electricity demanded (or perhaps apparent electricity consumption, denoted $D$ ) and the amount of electricity consumed (or actual electricity consumption, denoted $C$ ). The former measures the consumption of electricity-consuming services, rather than the consumption of electricity per se, and affects assessments of comfort and environmental impact. The latter measures the actual amount of electricity consumed, and affects assessments of cost. With the introduction of an efficiency intervention using $(100 \times s) \%$ as much electricity as an old technology, the amount of electricity consumed is $C=s D$.

Efficiency interventions have the additional complexity that the service provided by the new technology might often not be precisely the same as previously experienced. There may also be teething problems upon the introduction of new technologies as users become accustomed to technologies that have remained the same for many years, with outright rejection and subsequent reversion to old technology being a possibility for some consumers. Levels of comfort are therefore given by $f_{2}(C)=p D$, where the new technology is judged to offer a service only $(100 \times p) \%$ as good as the previous technology. Efficiency interventions also affect assessments of cost, since less electricity is being used to provide the same level of service. Currently incurred cost is given by $f_{1}(C)=(1+t) r s D$. 


\subsubsection{Information interventions}

Information interventions operate by exerting an influence on the relative importance (i.e. the weights $w_{j}$ ) attached to the three objectives rather than the current evaluation of impact (so $f_{3}(C)=C$ ). The effect on the objectives is likely to depend on the type of intervention used. For example, feedback contrasting household electricity spending with historical figures or neighbourhood norms may increase the relative importance of the cost objective. More overt campaigns highlighting the damage caused by wasteful consumption may have the effect of increasing the weight associated with the impact goal at the expense of the weights for the other two objectives. We therefore allow information interventions to change any of a household's weights (changes in goals may also be modelled, but that is not done here), but only to a limited degree.

A maximum change is specified for the weight on the cost and environmental impact objective (weights sum to one, so that the comfort weight is indirectly influenced by changes to the other two). This maximum change essentially captures the salience of the information campaign through its ability to change a household's perceptions. While an information campaign is running, a household's weights will slowly change, from the initial weights, up to the maximum allowable change (provided that the intervention runs for long enough). Once an intervention ceases, the preferences start to fall back to their initial values. Exponential smoothing, which results in the decreasing marginal changes as the time following the introduction (or ceasing) of an intervention elapses, is used to model these changes.

Assessments of current cost $f_{1}(C)$, comfort $f_{2}(C)$, and impact $f_{3}(C)$ may be substituted into earlier expressions to find $\delta_{j}$, which may, in turn, be substituted into the basic equilibrium equality. A household's choice of how much electricity to use is therefore guided by the selection of a demand level $D$ satisfying

$$
\begin{aligned}
w_{\text {comf }}\left(\max \left\{g_{\text {comf }}-p D, 0\right\}\right)= & w_{\text {cost }}\left(\max \left\{(1+t) r s D-g_{\text {cost }}, 0\right\}\right) \\
& +w_{\text {imp }}\left(\max \left\{D-g_{\text {imp }}, 0\right\}\right) .
\end{aligned}
$$

\subsection{Additional model features}

In addition to the basic consumption model and models of the three intervention programs described above, a number of additional features were included in the full model, but at this stage do not play a role in any of the results, because they are held constant or set to null values. Specific features include: households only becoming aware of their spending periodically (i.e. through some billing mechanism), with the inter-invoice period potentially affected by an intervention; capacity for increases in a particular household's electricity demand being limited (for example, by the number of electricity-consuming devices owned) and the determination of this capacity for consumption itself being an outcome of the decision-making process. The intention is that, when the basic model is validated against further empirical data, these additional elements may be useful as a means of fine-tuning the basic model. 


\section{Model results and formulation of hypotheses}

Our primary aim is to use the system dynamics model to develop qualitative hypotheses relating responses to efficiency interventions to household attitudes. We do this by exploring the possible effects of different intervention strategies on the consumption of electricity for four household 'types' which differ broadly in their attitudes. Although the model returns a quantitative output, our view is that because of the difficulty of reliably measuring attitudinal inputs like goals and weights in a short survey, comparisons between survey and model results should be based on qualitative rather than quantitative hypotheses. This means that the precise values used to model the simulated interventions and household types are less important than the general characteristics which they impart. We performed sensitivity analyses around each of the values used to establish that our hypotheses and conclusions are not sensitive to the precise values used.

The simulated interventions are:

1. No intervention (baseline).

2. A $10 \%$ increase in the per-unit price of electricity.

3. A $10 \%$ efficiency saving (with no dissatisfaction with the new technology).

4. A $10 \%$ efficiency saving (with satisfaction with the new technology $90 \%$ of the old).

5. A 'feedback'-type awareness campaign: this has the effect of increasing the weight allocated to the cost objective by $10 \%$.

6. A 'greening'-type awareness campaign: this has the effect of increasing the weight allocated to the environmental impact objective by $10 \%$.

7. A joint price and efficiency (no dissatisfaction) intervention (interventions 2 and 3 together).

8. A joint price and 'feedback' information intervention (interventions 2 and 5 together).

9. A joint price and 'greening' information intervention (interventions 2 and 6 together).

10. A joint efficiency (no dissatisfaction) and 'feedback' information intervention (interventions 3 and 5 together).

11. A joint efficiency (no dissatisfaction) and 'greening' information intervention (interventions 3 and 6 together).

The four different household types are ones in which:

1. Cost is viewed as relatively more important than comfort, with no importance attached to environmental impact $\left(w_{\text {cost }}=0.7, w_{\text {comf }}=0.3\right.$ and $\left.w_{i m p}=0\right)$.

2. Cost is viewed as relatively more important than comfort, with a small but non-zero weight for environmental impact $\left(w_{\text {cost }}=0.7, w_{\text {comf }}=0.2\right.$ and $\left.w_{i m p}=0.1\right)$.

3. Cost is viewed as relatively more important than comfort, with no importance attached to environmental impact $\left(w_{\text {cost }}=0.3, w_{\text {comf }}=0.7\right.$ and $\left.w_{i m p}=0\right)$.

4. Cost is viewed as relatively more important than comfort, with a small but non-zero weight for environmental impact $\left(w_{\text {cost }}=0.3, w_{\text {comf }}=0.6\right.$ and $\left.w_{\text {imp }}=0.1\right)$.

The same goals $\left(g_{\text {cost }}=60, g_{\text {comf }}=90\right.$ and $\left.g_{i m p}=30\right)$ are used for each household; because all results are to be expressed in relative terms (i.e. percentage changes), the 
actual units of measurement are not important, and have been expressed on a scale from 0 to 100. The magnitudes of the goals affect the equilibrium consumption level but do not influence the relative effects of the interventions.

The relative effects of each intervention on the amount of electricity consumed are shown in Table 1. Because of the distinction between electricity consumed (essentially, the amount of electricity required from a supplier in order to perform certain activities) and electricity demanded (a measure of the number/type of activities performed), the relative effects of interventions on electricity demanded are also shown in Table 1, in parentheses. These and other exploratory results formed the basis for a number of discussions with a group of energy researchers also working on the project. The purpose of these discussions was to establish whether the results of the model were consistent with existing knowledge, to identify any problem areas in the model, and to use the model to generate new insights into potential responses to interventions.

As examples, we consider the rebound effects observed for both the price and efficiency interventions. According to the model, the reason underlying rebound responses to pricebased interventions is that price interventions increase the gap between the cost goal and the currently incurred cost, while leaving the gap between the comfort goal and current comfort unchanged. This provides an incentive to immediately decrease the demand for electricity, while the comfort goal acts as a counterweight to this incentive and induces some resistance to change. The model is thus able to exhibit known empirical behaviour (analogous to demand-price elasticity) while providing a parsimonious explanation for that behaviour.

More importantly, the model also suggests results that (to our knowledge) have not been shown empirically. It can be shown that in order to restore a balance between objectives following a price intervention, demand must decrease until the ratio of new to old demand is given by $\left(w_{\text {comf }}+(1+t) w_{\text {cost }}+w_{i m p}\right)^{-1}$, provided that the change in demand does not result in any of the goals being fully achieved. The expression simplifies further to $\left(1+t w_{\text {cost }}\right)^{-1}$, because of the constraint that weights must sum to one. Therefore, households that place a greater importance on cost relative to comfort will experience larger decreases in electricity demand following price interventions, which is why the simulated rebound is greater in households placing more weight on the comfort objective and is unaffected by the environmental impact weight (which we increased at the expense of comfort weight, leaving $w_{\text {cost }}$ unchanged). The model suggests that increasing $w_{\text {imp }}$ at the expense of $w_{\text {cost }}$ would reduce the impact made by price-based interventions. This suggests the following two research hypotheses:

Hypothesis 1: For price interventions, greater rebound effects are experienced in households for whom cost is relatively unimportant.

Hypothesis 2: Increasing the importance of the impact objective at the expense of the cost objective would reduce the impact made by price-based interventions.

In contrast the reason for rebound responses to efficiency interventions is that the efficiency saving decreases the gap between the cost goal and the current cost, while leaving the comfort gap unchanged. This provides an incentive to increase the amount of electricity demanded. It can be shown that in the case of satisfying efficiency interven- 


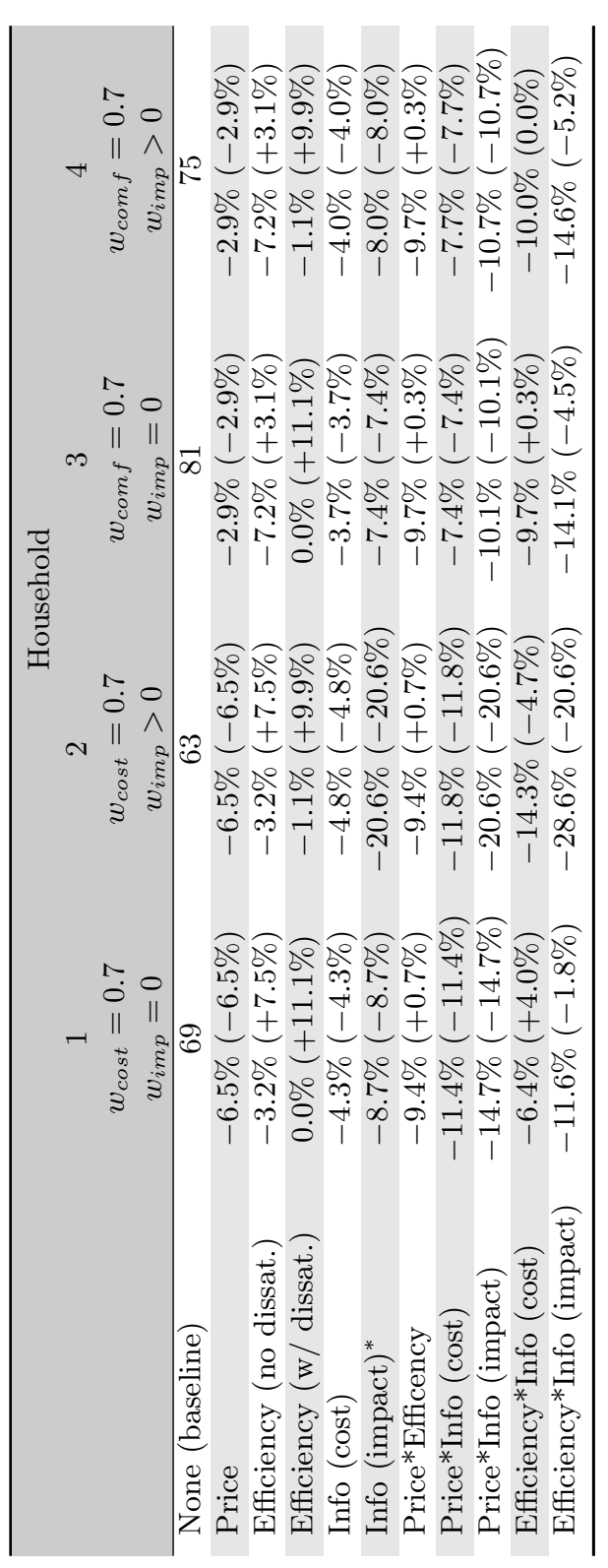

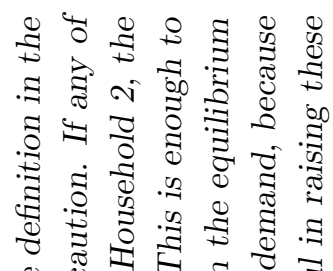

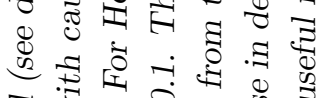

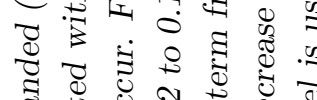

สี

ช

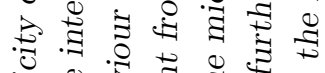

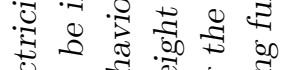

จ

फ

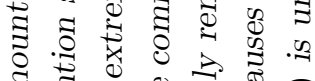

สี

ปี

สี

芯

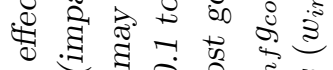

击

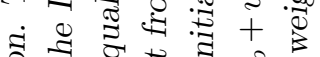

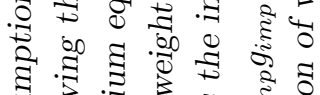

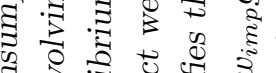

व $:$ : $:$ :

ง

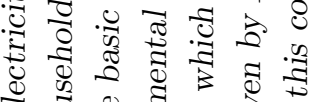

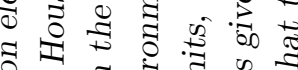

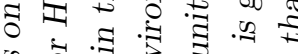

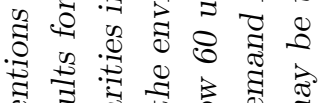

สี

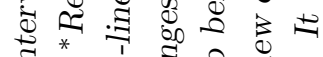

. * $*$ สี कि

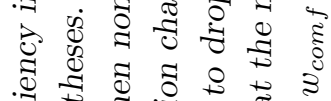

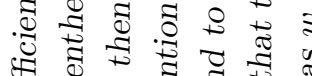

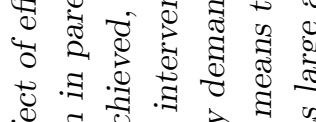

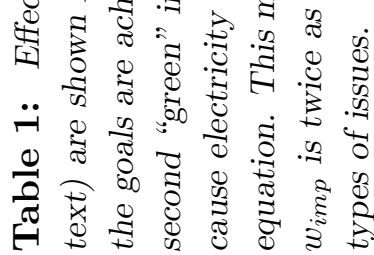


tions, a balance between objectives will be restored when demand rises by a factor of $\left(w_{\text {comf }}+s w_{\text {cost }}+w_{\text {imp }}\right)^{-1}$, which simplifies to $\left[1-(1-s) w_{\text {cost }}\right]^{-1}$. This factor increases in $w_{\text {cost }}$, suggesting that households that place a greater importance on cost relative to comfort will experience greater increases in demand. This result is the opposite of that obtained for price interventions, and can be expressed in the form of the following hypothesis:

Hypothesis 3: For efficiency interventions, greater rebound effects are experienced in households for whom cost is relatively important.

Through discussion and reasoning through the model in a similar manner to that just described, a number of further hypotheses were established. These are listed without further motivation below:

Hypothesis 4: Greater rebound effects are experienced if an efficiency intervention involves a technology that does not deliver as satisfying a service as before. If the new technology is more satisfying, negative rebound is possible.

Hypothesis 5: Rebound effects occurring as a result of unsatisfactory new technology decrease as more weight is placed on environmental issues.

The use of a literature survey of previous research in conjunction with discussion sessions to construct a preference-based system dynamics model of household electricity consumption, and the use of this model as a basis for generating further discussion around a set of testable hypotheses, forms what came to be known as the 'first phase' of the project.

\section{$6 \quad$ Integrating survey data into the system dynamics model}

In the 'second phase' of the modelling approach to be described in this section, data collected from an actual intervention are used to test several of the hypotheses above, with a view to evaluating the model and refining it further should this prove necessary.

\subsection{Survey details}

The data used for testing the model come from an observational study involving a free (to the consumer) CFL light bulb exchange program conducted in Prince Albert, a town in the Western Cape province of South Africa. The exchange is part of the South African utility provider Eskom's national Demand Side Management (DSM) programme. Prince Albert was chosen as a study site due to its relative isolation, and since it was one of the only remaining towns that had not yet seen a CFL exchange, so that baseline consumption and survey data could be collected. The timing of the intervention was also known. The town contains a mix of high and low-income households that have historically been separated. Most of the households rely on electricity for all end-uses with some of the wealthier households having access to alternative energy sources (solar water heating, photovoltaics and LPG) while the poorer households derive some of their heating requirements from wood fuel when money for electricity runs out.

In June 2008, all households in Prince Albert participated in a program in which they were 
offered free CFLs in exchanged for their regular (incandescent) light bulbs. CFLs consume an average of $20 \%$ of the electricity that incandescent bulbs do. The data collected and prepared consists of electricity payment records from 1 November 2007 to 31 October 2009, and a panel of surveys that took place in June 2008, September 2008 and September 2009. Consumption and survey data are available for a total of 122 households. All households included in our study are on a 'pre-paid' electricity system, i.e. when they wish to purchase electricity they do so by purchasing a number of units (determined by the household) from vendors. Some households qualify for a free monthly quota of electricity ('free basic units'), which are also recorded at the time of a first purchase in a particular month. The payment and free electricity records have been used to estimate a monthly electricity consumption figure for each household, expressed as the average number of units of electricity consumed per day over the course of each month.

The final survey conducted in September 2009 covered a range of issues relating to the intervention and energy usage in general ${ }^{5}$. Our analysis makes use of a small part of the survey capturing household preferences for different aspects of electricity consumption and satisfaction with the CFL light bulbs. Households were asked to rate how important each of the factors 'cost of electricity', 'usefulness and enjoyment' (comfort), and 'impact on environment' were in their decisions of how much electricity to consume prior to the intervention. Responses were recorded on a scale from 1 (not important at all) to 10 (very important). These responses were scaled to sum to one within each household in order to provide estimates of weights on the cost, comfort, and impact objectives respectively. While this is admittedly a fairly crude form of importance weight measurement, a more sophisticated measurement brings its own problems - specifically stronger user-involvement and greater surveyor training. This, in conjunction with an already lengthy survey and the fact that our work formed a relatively minor part thereof, motivated the use of a simple and direct measurement question. Satisfaction with the intervention was measured by asking respondents to rate the effect of the intervention on the following five aspects, on a scale from 1 (very negative effect) to 10 (very positive effect): money saved on electricity; ability to use lights; quality of light in my home; my responsibility for the environment; my awareness of energy matters. An overall satisfaction score was calculated by taking the average rating over these five questions.

In addition to the efficiency (CFL exchange) intervention, there were two nationwide increases in the price of electricity, on 1 July 2008 and 1 July 2009. The exact magnitude of the increase for a household is fairly complicated and depends on the tariff category (which, in turn, depends on historical usage) a household falls into, but were of the order of $25 \%$ and $35 \%$, respectively. Because the data captures monetary spend as well as units consumed, it was possible to calculate relative price increases for each household over time.

\subsection{Tests of research hypotheses}

Our research hypotheses (stated in Section 5) can be expressed in statistical terms and tested via formal hypothesis testing. All hypotheses are tested by means of linear re-

\footnotetext{
${ }^{5}$ Two earlier surveys were also conducted, in June 2008 and September 2008. Neither of these surveys contained questions regarding preferences for different aspects of electricity consumption or satisfaction with the intervention. We therefore do not make use of them here.
} 
gression models using a random intercept to account for between-household heterogeneity in electricity consumption. The dependent variable in all cases is the natural logarithm of consumption, which transforms the outcome to one that is normally distributed. The independent variables that are included in the model differ depending on the hypothesis to be tested. The models for the various hypotheses are as follows:

Hyp 1: $\ln \left(C_{i t}\right)=\beta_{0}+\beta_{1} P C_{i t}+\beta_{2} w_{\text {cost }, i}+\beta_{3} P C_{i t} w_{\text {cost }, i}+\mu_{i}+\epsilon_{i t}$,

Hyp 2: $\ln \left(C_{i t}\right)=\beta_{0}+\beta_{1} P C_{i t}+\beta_{2}\left(w_{i m p, i}-w_{\text {cost }, i}\right)+\beta_{3} P C_{i t}\left(w_{i m p, i}-w_{\text {cost }, i}\right)+\mu_{i}+\epsilon_{i t}$,

Hyp 3: $\ln \left(C_{i t}\right)=\beta_{0}+\beta_{1} E I_{i t}+\beta_{2} P C_{i t}+\beta_{3} w_{\text {cost }, i}+\beta_{4} E I_{i t} w_{\text {cost }, i}+\mu_{i}+\epsilon_{i t}$,

Hyp 4: $\ln \left(C_{i t}\right)=\beta_{0}+\beta_{1} E I_{i t}+\beta_{2} P C_{i t}+\beta_{3} s a t_{i t}+\mu_{i}+\epsilon_{i t}$,

Hyp 5: $\ln \left(C_{i t}\right)=\beta_{0}+\beta_{1} E I_{i t}+\beta_{2} P C_{i t}+\beta_{3} w_{i m p, i}+\beta_{4} E I_{i t} w_{c o s t, i}+\mu_{i}+\epsilon_{i t}$,

where $i$ indexes household and $t$ indexes time/month, $C_{i t}$ denotes the average daily consumption by household $i$ during month $t, P C_{i t}$ denotes the price change (as a percentage of the original baseline price), $E I_{i t}$ denotes an indicator for efficiency intervention, $w_{c o s t, i}$ denotes an importance weight for the cost objective, $w_{i m p, i}$ denotes an importance weight for the impact objective, $s a t_{i t}$ denotes satisfaction with an intervention (set to zero prior to the intervention), $\mu_{i}$ denotes the random effect for household $i$ distributed $N\left(0, \sigma_{\mu}^{2}\right)$, and $\epsilon_{i t}$ denotes the random error term distributed $N\left(0, \sigma_{\epsilon}^{2}\right)$.

Different sub-samples of time or households are used for some of the hypotheses. Hypotheses 1 and 2 refer to the impact of price-interventions only. As a result, attention is confined to the months after the CFL exchange in June 2008 - considering months prior to this period would confound the effect of price and efficiency interventions. To account for seasonal effects, consumption in July to October 2008 (a 'before' period) and July to October 2009 (a period 'after' the second price increase) was considered. This allows for the testing of the impact of a price increase independent of the earlier efficiency intervention. Hypotheses 3 and 4 are tested using the full sample. Hypothesis 5 refers to rebound effects occurring as a result of unsatisfying new technology. Attention is restricted to those households giving relatively poor satisfaction ratings to the exchanged CFLs. A cut-off of 6 (out of 10) was used, which reduces the sample to the 21 (out of the total 122) most dissatisfied households.

Results of the hypothesis tests are shown in Table 2. In each column the variable to which the hypothesis applies is stated, and the direction of the effect suggested by the system dynamics model is given. Regression coefficients for all effects in each regression model are given, with standard errors indicated in parentheses. The parameter estimate corresponding to the variable of interest for each hypothesis test is highlighted in boldface, and $p$-values associated with each (one-sided) hypothesis test are given in the final row of the table. Hypotheses 1, 2, 3 and 5 are all strongly supported by the data, and there is weak support for hypothesis 4 .

\footnotetext{
${ }^{6}$ The coefficient for $w_{i m p, i}$ is the same magnitude but oppositely signed to the coefficient for $w_{\text {cost }, i}$ because a single coefficient is estimated for the difference $w_{i m p, i}-w_{c o s t, i}$ (see the model formulation). The same will be true of the two interaction terms involving $P C_{i t}$.
} 


\begin{tabular}{|c|c|c|c|c|c|}
\hline $\begin{array}{l}\text { Variable } \\
\text { Hypothesis } \\
H_{0} \text { (Null) } \\
H_{1} \text { (Model) }\end{array}$ & $\begin{array}{c}\text { Hyp } 1 \\
P C_{i t} w_{\text {cost }, i} \\
\beta \geq 0 \\
\beta<0\end{array}$ & $\begin{array}{c}\text { Hyp } 2 \\
P C_{i t} w_{i m p, i} \\
\beta \leq 0 \\
\beta>0\end{array}$ & $\begin{array}{c}\text { Hyp } 3 \\
E I_{i t} w_{\text {cost }, i} \\
\beta \leq 0 \\
\beta>0\end{array}$ & $\begin{array}{l}\text { Hyp } 4 \\
\text { sat }_{i t} \\
\beta \geq 0 \\
\beta>0\end{array}$ & $\begin{array}{c}\text { Hyp } 5 \\
E I_{i t} w_{i m p, i} \\
\beta \leq 0 \\
\beta>0\end{array}$ \\
\hline$P C_{i t}$ & $0.10(0.16)$ & $-0.21(0.04)$ & $-0.11(0.04)$ & $-0.10(0.04)$ & $0.15(0.11)$ \\
\hline$E I_{i t}$ & & & $-0.11(0.08)$ & $0.06(0.02)$ & $0.37(0.19)$ \\
\hline$w_{\text {cost }, i}$ & $-0.48(0.77)$ & $-0.50(0.46)$ & $-0.67(0.70)$ & & \\
\hline$w_{i m p, i}$ & & $-0.50^{6}(0.46)$ & & & $1.23(1.62)$ \\
\hline$s_{a t}$ & & & & $-0.02(0.02)$ & \\
\hline$P C_{i t} w_{\text {cost }, i}$ & $-1.01(0.43)$ & $-0.59(0.22)$ & & & \\
\hline$P C_{i t} w_{i m p, i}$ & & $0.59(0.22)$ & & & \\
\hline$E I_{i t} w_{\text {cost }, i}$ & & & $0.52(0.23)$ & & \\
\hline$E I_{i t} w_{i m p, i}$ & & & & & $-1.48(0.60)$ \\
\hline Constant & $2.13(0.26)$ & $2.01(0.23)$ & $2.01(0.23)$ & $1.80(0.06)$ & $1.41(0.53)$ \\
\hline$\sigma_{\mu}$ & 0.58 & 0.58 & 0.62 & 0.62 & 0.79 \\
\hline$\sigma_{\epsilon}$ & 0.28 & 0.28 & 0.43 & 0.43 & 0.54 \\
\hline$p$ for test & 0.009 & 0.003 & 0.012 & 0.110 & 0.007 \\
\hline
\end{tabular}

Table 2: Results of hypothesis tests for five hypotheses regarding changes in electricity consumption behaviour occurring in different household types in response to price and efficiency interventions.

\section{Discussion}

This section contains a discussion on our experience of modelling household responses for energy efficiency interventions as described in this paper.

\subsection{Use of empirical data to generate confidence in system dynamics models}

Following the hypothesis tests one further meeting took place to discuss the results and their implications for the final model. There was some surprise that the model had been well-supported by the data, given the complexity of the hypotheses (all but one involved interactions of some sort), the relatively crude approach used to measure preferences, and the amount of variability present in the consumption figures. The support provided by the data generated confidence in the underlying model and a sense that the model had been 'validated'. This led to greater acceptance of the model's structure (particularly the use of preferences for different household objectives that distinguish this model from many other energy models) and a greater willingness to explore and accept the other implications of the model (i.e. those that were not part of the hypothesis tests).

The role that data can play in inspiring a sense of confidence in a fairly qualitative system dynamics model such as ours was perhaps the most interesting aspect of our experience. Acceptance and trust of the final model by those involved in its construction is a basic requirement of a successful modelling process — validation as "the process of establishing confidence in the soundness and usefulness of a model" (Forrester and Senge, 1980). System dynamics practitioners often prefer more qualitative tests of validity to statistical tests (Forrester and Senge, 1980; Barlas, 1996; Coyle and Exelby, 2000), but our experience has been that a statistical comparison against empirical data (where possible 
and in conjunction with other checks) can be effective in achieving the acceptance of a model. The comparison need not be particularly detailed - in this application it was assessed whether the direction of a small number of non-trivial effects implied by the model matched those observed empirically. In fact, for the types of problems for which system dynamics (or even 'softer' OR) methods are usually applied, it would seem important not to base comparisons on overly detailed quantitative predictions of behaviour.

Ideally comparisons between an initial model and empirical data would be used as a complement to further discussion and modelling. One might expect differences between model output and empirical findings to provide a stimulus for further modelling by identifying behaviour that the model is unable to account for and areas of the model that are questionable. In the application considered here, no real discrepancies between model output and empirical data arose, and so further modelling was deemed, at this point in time, unnecessary. As further implications are explored and other data become available, this may change.

\subsection{Use of system dynamics to explore unusual points of view}

In our case, the focus on relatively abstract preferences for cost, comfort, and environmental impact constituted an 'unusual' point of view. In the analysis of energy systems, consumer response is usually captured through elasticity parameters derived from econometric models of energy demand in the economy, with little focus on the micro-level response. Attitudinal information, particularly regarding preferences, is relatively rare in energy research, although it is increasingly being advocated. One of the challenges facing those advocating attitudinal approaches is to motivate for the collection of such data, which tends to be expensive relative to the collection of behavioural data like electricity consumption, prices, etc. In our application system dynamics proved to be a valuable tool for generating such motivation, and it was only following the construction of the initial model that a decision was made to collect attitudinal data as part of the project. Given the usefulness of these data, the collection of attitudinal data may prove easier to motivate for in the future. Our experience suggests that the system dynamics approach is especially useful in cases where, for historical or economic reasons, advancing a particular point of view through traditional modelling approaches is difficult. This has some significance in developmental applications, where empirical data are not always readily available or are prohibitively expensive to collect.

\subsection{Importance of understanding attitudes and preferences underlying energy consumption}

From the perspective of energy research, a useful finding is how a simple model of household preferences is able to capture quite sophisticated dynamics in consumption, particularly in terms of responses to different intervention scenarios. Several researchers have called for more emphasis on psychological models of energy consumption, and for a greater accounting for the attitudes and decisions underlying energy-related behaviour rather than a direct focus on the behaviour (Swim et al., 2009; Griskevicius et al., 2008; Gyberg and Palm, 2009; Abrahamse et al., 2005; Abrahamse et al., 2007; Fischer, 2008; Owens 
and Drifill, 2008). The system dynamics model described above is largely based on this emerging psychology. The results provide support for the three primary criteria identified by most researchers as important determinants of energy consumption - labelled in this paper as cost, comfort, and impact objectives - and suggest that these three criteria, if not exhaustive, are at least sufficient to explain several observed responses to interventions, and together with the satisfaction derived from an intervention are able to collectively provide simple and quite intuitive explanations for several important aspects of rebound behaviour.

\section{Conclusion}

A case study of a modelling process undertaken in order to better understand how households might go about deciding (if only implicitly) how much electricity to consume was presented in this paper. No definitive model of household consumption exists, and there is still much that needs to be learned about the drivers of behaviour at the household level. System dynamics and soft OR methods are well suited to applications such as this, where the problem definition is somewhat vague and subject to differing interpretations, and a major aim of the process is to gain a better understanding of the system and what forms possible solutions might take. The approach that was followed may be summarised as follows:

1. Identify which aspects of attitude and behaviour have been found in the literature to affect household electricity consumption.

2. Integrate these findings into a single conceptual model of household electricity consumption, using system dynamics as an aid to the construction of such a model.

3. Generate a small number of household types (with different preferences) and intervention scenarios, and use the model to evaluate the potential impact of each intervention scenario on each household. Use these results as a basis for discussion.

4. Use the results obtained from the initial model to generate a small number of testable research hypotheses about consumption behaviour. These hypotheses related to how households endowed with different attitudinal characteristics might respond to a particular intervention.

5. Use survey data to evaluate the research hypotheses. Use the results of these hypothesis tests as a basis for further discussion and modification of the model, where necessary, until the stakeholders are satisfied that the aims of the modelling process have been achieved.

A summary of the authors' experience of applying the above process is that:

1. The inclusion of attitudinal elements (and, in particular, preferences for conflicting energy-related attributes) enabled quite sophisticated dynamics in electricity consumption to be captured, in terms of responses to different intervention scenarios, and to provide fairly simple explanations for this behaviour.

2. A soft OR approach (in this instance, system dynamics) proved useful in exploring the behaviour of a complex system which is as yet not well understood, and for which comprehensive data are not readily available. 
3. The main benefits of using such an approach were those that are usually claimed: that it provided a basis for structuring the collective thoughts and ideas of a group of stakeholders, and for integrating different information on various parts of the system.

4. Although a benefit of the soft OR approach is that it allows one to explore the impact of potential scenarios on the system, in application areas where modelling has traditionally been predominantly quantitative or where an unusual point-of-view of the system is taken, confidence in the extrapolations may be limited.

5. The use of empirical data to test a small number of relatively simple but non-trivial predictions made by the model was an effective tool in reducing skepticism around the model. Ideally, differences between model output and empirical findings should be used to stimulate further discussion and modelling modification by identifying behaviour which the model is unable to account for and areas of the model that are questionable.

The model presented here provides a useful basic model for the preferential and other attitudinal drivers underlying electricity consumption. There remain a number of more sophisticated elements of consumption behaviour that may be included as a means of finetuning the basic model. We have mentioned a small number of these: different invoicing mechanisms, the determination of capacity constraints on consumption, and varying the length and frequency of information campaigns. As the literature around energy consumption grows, it will likely become possible to develop more comprehensive models of consumption capable of explaining some of the more subtle aspects of behaviour. Our results suggest that attitudinal elements may be expected to play an important role in such models.

\section{References}

[1] Abrahamse W, Steg L, Vlek C \& Rothengatter T, 2005, Review of intervention studies aimed at household energy conservation, Journal of Environmental Psychology, 27, pp. 273-291.

[2] Abrahamse W, Steg L, Vlek C \& Rothengatter T, 2007, The effect of tailored information, goal setting and tailored feedback on household energy use, energy-related behaviours and behavioural antecedents, Journal of Environmental Psychology, 27, pp. 265-276.

[3] Barlas Y, 1996, Formal aspects of model validity and validation in system dynamics, System Dynamics Review, 12(3), pp. 183-210.

[4] Barker T, Dagoumas A \& Rubin J, 2009, The macroeconomic rebound effect and the world economy, Unpublished Report, Cambridge Centre for Climate Change Mitigation Research, University of Cambridge.

[5] BARker T, Ekins P \& Foxon T, 2007, The macro-economic rebound effect and the UK economy, Energy Policy, 35, pp. 4935-4946.

[6] Berkhout PHG, Muskens JC \& Velthuijsen JW, 2000, Defining the rebound effect, Energy Policy, 28, pp. 425-432.

[7] Birol F \& Keppler JH, 2000, Prices, technology development and the rebound effect, Energy Policy, 28, pp. 457-469.

[8] Bladh M \& Krantz H, 2008, Towards a bright future? Household use of electric light: A microlevel study, Energy Policy, 36, pp. 3521-3530.

[9] Caird S, Roy R \& Herring H, 2008, Improving the energy performance of UK households: Results from surveys of consumer adoption and use of low- and zero-carbon technologies, Energy Efficiency, 1, pp. 149-166. 
[10] Coyle G \& Exelby D, 2000, The validation of commercial system dynamics models, System Dynamics Review, 16(1), pp. 27-41.

[11] Dyner I, Smith RA \& PEÑA GE, 1995, Systems dynamics modelling for residential energy efficiency analysis and management, Journal of the Operational Research Society, 46, pp. 1163-1173.

[12] EK K \& SöDerholm P, 2010, The devil is in the detail: Household electricity saving behaviour and the role of information, Energy Policy, 38, pp. 1578-1587.

[13] Fischer C, 2008, Feedback on household electricity consumption: A tool for saving energy, Energy Efficiency, 1, pp. 79-104.

[14] Ford F, 1999, Modeling the environment: An introduction to system dynamics models of environmental systems, Island Press, Washington (DC).

[15] Forrester J \& Senge P, 1980, Tests for building confidence in system dynamics models, TIMS Studies in the Management Sciences, 14, pp. 209-228.

[16] Grepperud S \& Rasmussen I, 2004, A general equilibrium assessment of rebound effects, Energy economics, 26, pp. 261-282.

[17] Griskevicius V, Cialdini R \& Goldstein, 2008, Social Norms: An underestimated and underemployed lever for managing climate change, International Journal of Sustainability Communication, 3, pp. 5-13.

[18] Gyberg P \& Palm J, 2009, Influencing households' energy behaviour - How is this done and on what premises?, Energy Policy, 37, pp. 2807-2813.

[19] JaCCARD M \& Bataille C, 2000, Estimating future elasticities for the rebound effect, Energy Policy, 28, pp. $451-455$.

[20] Laitner JAS, 2000, Energy efficiency: Rebounding to a sound analytical perspective, Energy Policy, 28, pp. $471-475$.

[21] Levine M, Geller H, Koomey J, Nadel S \& Price L, n.d., Electricity end-use efficiency: experience with technologies, markets and policies throughout the world, American Council for an EnergyEfficient Economy, [Online], [Accessed October 28 ${ }^{\text {th }}$ 2010], Available from http://www.aceee.org/ pubs/i921.htm.

[22] Morecroft J, 2007, Strategic modelling and business dynamics: A feedback systems approach, John Wiley \& Sons, Hoboken (NJ).

[23] Mehlwana A \& QASE N, 1996, Social determinants of energy use in low-income metropolitan households in the Western Cape, Unpublished Report, Energy for Development Research Centre, University of Cape Town, Cape Town.

[24] NässÉn J \& Holmberg J, 2009, Quantifying the rebound effects of energy efficiency improvements and energy conserving behaviour in Sweden, Energy Efficiency, 2, pp. 221-223.

[25] Owens S \& Driffill L, 2008, How to change attitudes and behaviours in the context of energy, Energy Policy, 36, pp. 4412-4418.

[26] Roy J, 2000, The rebound effect: Some empirical evidence from India, Energy Policy, 28, pp. 433438.

[27] SAunders HD, 2000, A view from the macro side: Rebound, backfire and Khazzoom-Brookes, Energy Policy, 28, pp. 439-449.

[28] Sorrell S, Dimitropoulos J \& Sommerville M, 2009, Empirical estimates of the direct rebound effect: A review, Energy Policy, 37, pp. 1356-1371.

[29] Swim J, Clayton S, Doherty T, Gifford R, Howard G, Reser J, Stern P \& Weber E, 2009, Psychology and global climate change: Addressing a multi-faceted phenomenon and set of challenges, Report by the American Psychological Association's Task Force on the Interface Between Psychology and Global Climate Change, [Online], [Accessed August $1^{\text {st }}$ 2010], Available from http://www.apa. org/science/climate-change.

[30] Vensim, 2010, Seeing simulation with synthesim, [Online], [Cited November $7^{\text {th }}$ 2010], Available from http://www. vensim.com.

[31] Wall R \& Crosbie T, 2009, Potential for reducing electricity demand for lighting in households: An exploratory socio-technical study, Energy Policy, 37(3), pp. 1021-1031. 